\title{
Die Teichräumung im Botanischen Garten schafft Platz für Nymphaea alba
}

\author{
Elke Brude, Andreas König, Kai Uwe Nierbauer \& Hilke Steinecke
}

\begin{abstract}
During the winter 2018/2019, the pond of the Botanical Garden Frankfurt was completely desludged. For this purpose, all water lily hybrids (Nymphaea odorata $\times$ alba) had to be removed. Instead of this hybrids, the white water lily $($ Nymphaea alba) will be planted next year. In Hesse, this species is rare and endangered.
\end{abstract}

\section{Zusammenfassung}

Im Winter 2018/2019 wurde der große Teich im Botanischen Garten Frankfurt abgelassen und entschlammt. Die Hybrid-Seerosen (Nymphaea odorata $\times$ alba) wurden entfernt, um Platz für eine Erhaltungskultur der in Hessen seltenen und vom Aussterben bedrohten Weißen Seerose (Nymphaea alba) zu schaffen.

\section{Reges Leben am Teich}

Der Sitzplatz am Teich im Botanischen Garten ist bei vielen Besucherinnen und Besuchern sehr beliebt. Von hier aus kann man vor allem im Frühjahr die vielen Wasserfrösche gut beobachten, die bis in den Sommer ein laut keckerndes Froschkonzert anstimmen. Zudem schwirren auch immer wieder verschiedene Groß- und Kleinlibellen über die Wasseroberfläche. Manche bilden gerade Paarungsräder oder tauchen ihren Hinterleib ins Wasser, um ihre Eier abzulegen. Das mesotrophe Gewässer ist immer dicht mit submersen Wasserpflanzen bewachsen, zu nennen wären hier vor allem das Haarblättrige Laichkraut (Potamogeton trichoides), die Armleuchteralge (Chara vulgaris), das Ährige Tausendblatt (Myriophyllum spicatum) und das Raue Hornblatt (Ceratophyllum demer-

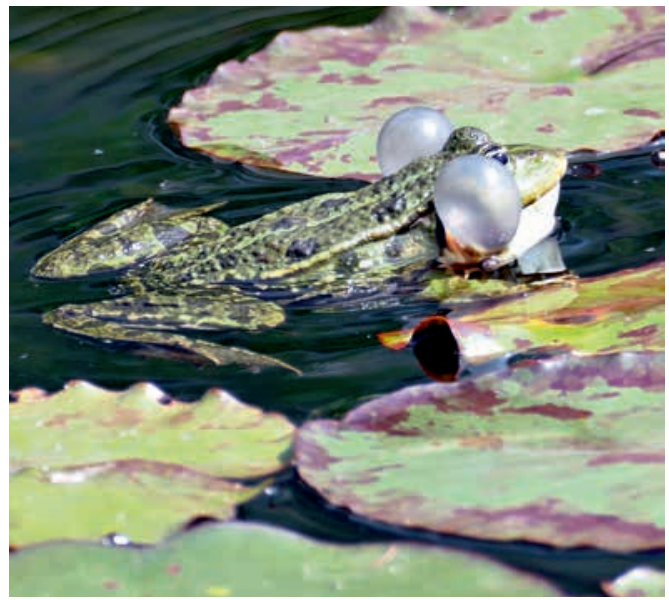

Abb. 1: Im Teich leben zahlreiche Wasserfrösche (Pelophylax). (Foto: H. STEINECKe) sum). Auf der Wasseroberfläche schwimmen die Blätter von See- und Teichrosen (Nymphaea sp., Nuphar lutea) und im Sommer erscheinen deren Blüten. Bei den Seerosen handelte es sich bisher um Hybrid-Seerosen (Nymphaea odorata $\times$ alba, vgl. Nierbauer 2014), in Zukunft soll es die in Hessen seltene und vom Aussterben bedrohte Weiße Seerose (Nymphaea alba) sein.

\section{Entschlammung im Winter 2018/2019}

Ein Teich bedarf der Pflege, damit er nicht verlandet. Die kleinen Bachläufe führen feine Sedimente herbei und aus dem benachbarten Buchenwald wird herbstliches Falllaub eingetragen. Vor allem bilden aber die vielen Wasserpflanzen und Tiere eine sich zersetzende Biomasse, die sich fortwährend als Detritus am Grund absetzt. Es ist immer

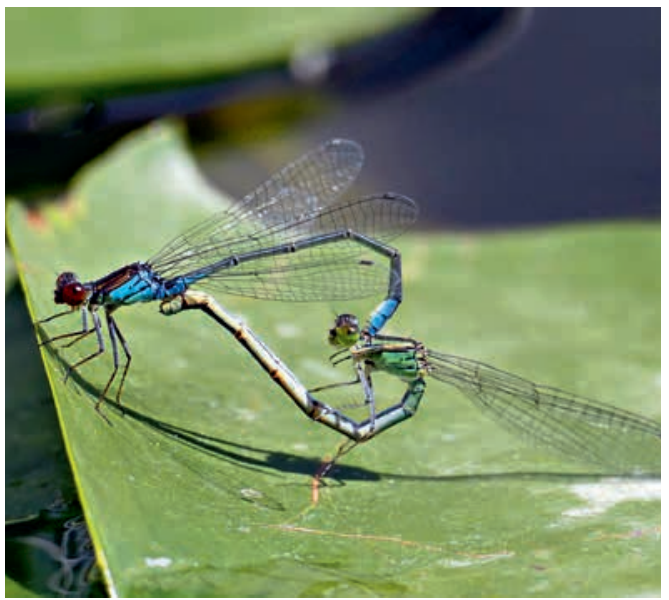

Abb. 2: Herzförmiges Paarungsrad des Kleinen Granatauges (Erythromma viridulum). (Foto: H. STEINECKe) 


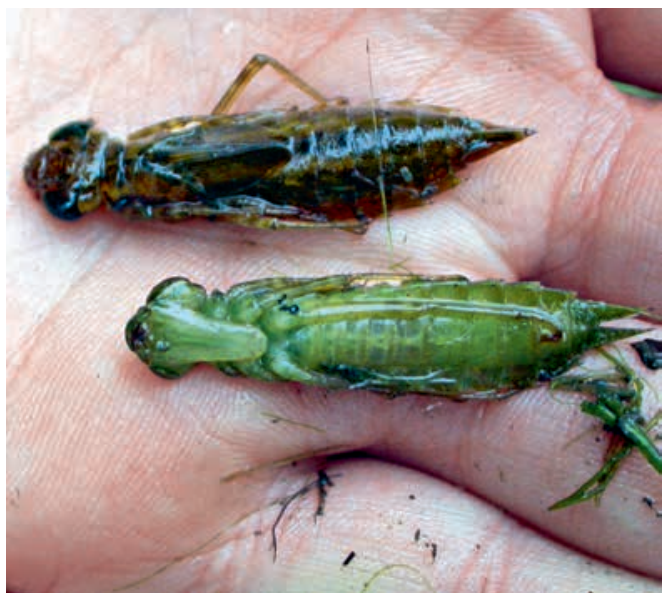

Abb. 3: Zwei aus dem Teich geborgene Großlibellenlarven für die Zwischenhälterung. (Foto: E. BRUdE)

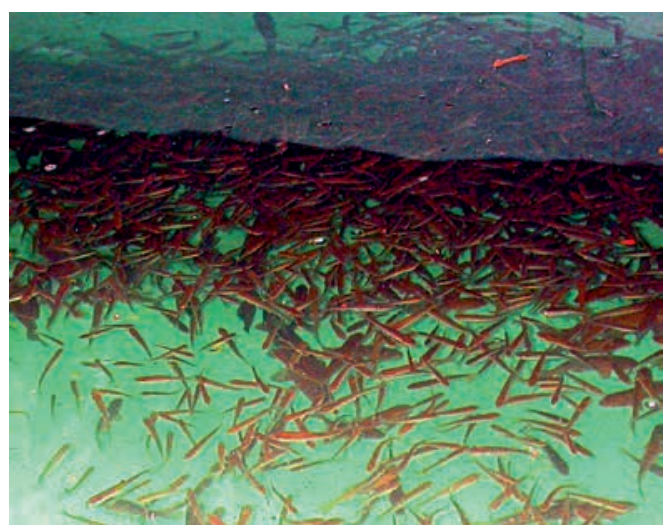

Abb. 4: Die Moderlieschen aus dem Teich werden in einem Container zwischengehältert. (Foto: E. BRUDE)

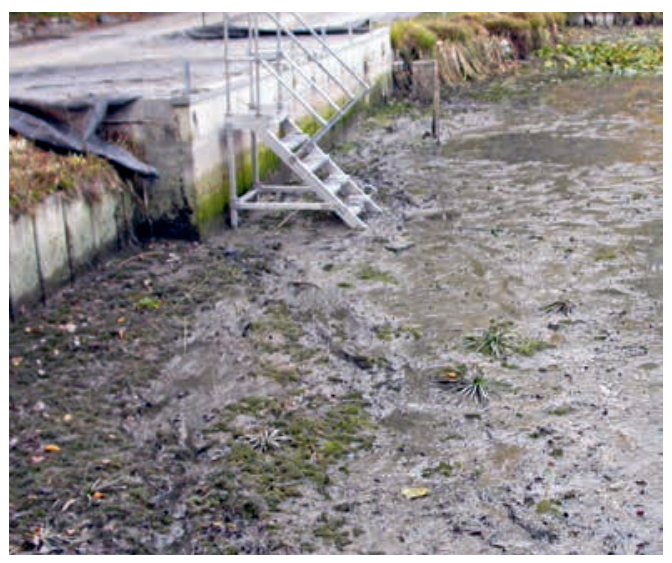

Abb. 5: Mobile Treppe für den schonenden und sicheren Ein- und Ausstieg. (Foto: E. Brude) wieder beeindruckend, wie viel Schlamm jedes Jahr aus dem Teich gepumpt werden kann.

Eine komplette Sanierung ist sehr aufwändig, zumal die im Teich lebenden Tiere erhalten werden sollen. Sie müssen abgefangen und gehältert werden. Die Teichentschlammung 2018 wurde ausführlich fotografisch dokumentiert.

Der Teich fasst bei Mittelwasser ca. $1000 \mathrm{~m}^{3}$. Das Ablassen des Teiches dauerte etwa vier Tage, in denen der Auslauf mit Schattennetzen für die Fische versperrt wurde. In den Restpfützen wurden dann von einem Fachmann alle Fische (Moderlieschen und Schleien) mit Genehmigung der Oberen Fischereibehörde abgefischt und bis zur Wiederbefüllung in einem großen Regenwasserbecken gehältert.

Was an Libellenlarven krabbelnd auf der Oberfläche entdeckt wurde, kam ebenfalls in Extrabehälter und wurde später zurückgesetzt.

Gewundert hatten wir uns, dass bei den Arbeiten im November praktisch keine der im Sommer zahlreichen Amphibien gesehen wurden. Wir hatten befürchtet, dass diese Tiere auf dem Teichboden überwintern und dann erst geborgen werden müssten, bevor mit Fahrzeugen gearbeitet werden kann. Die Amphibien überwintern aber offenbar in einem aus Richtung Grüneburgpark kommenden Drainagerohr. Ein Frosch schaute dort heraus, während im Teich selbst überhaupt keine Überwinterer beobachtet wurden. Nur einmal wurden zwei aktive Frösche gesehen.

Für den sicheren Ein- und Ausstieg und zur Schonung der Uferröhrichte während der rund vierwöchigen Teicharbeiten wurde eine mobile Metalltreppe an der Betonwand beim Unterstand aufgestellt.

Das Absaugen der zuvor zu Haufen zusammengetragenen Wasserpflanzen (vor allem Armleuchteralge, Hornblatt) und der flüssigen Schlammfraktion erfolgte mit dem Saugwagen einer Spezialfirma. 


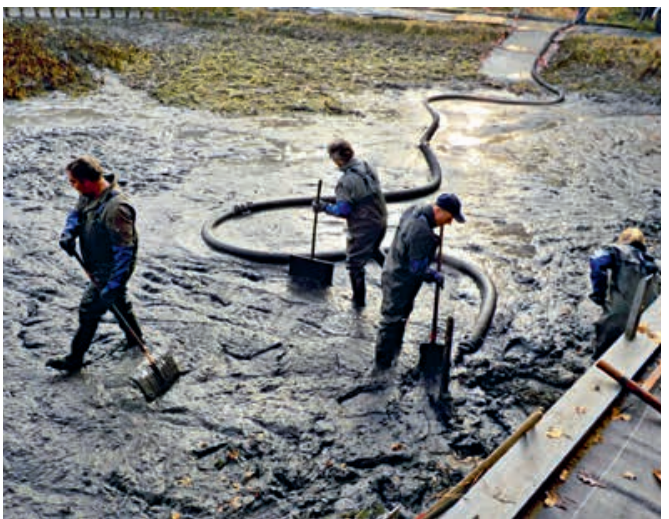

Abb. 6: Pflanzenreste und Schlamm werden mit Schneeschiebern vor die Schlauchöffnung geschoben und in den Wagen gesaugt. (Foto: E. BRUDE)

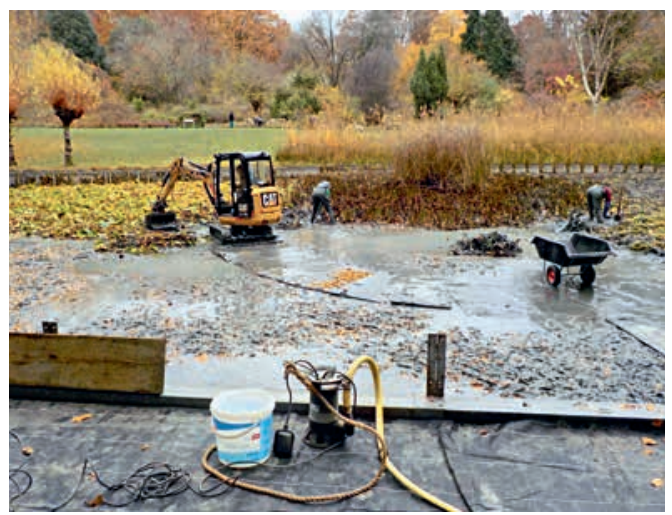

Abb. 8: Die Seerosen-Hybriden werden mit Hilfe eines Kleinbaggers entfernt. (Foto: E. BRUdE)

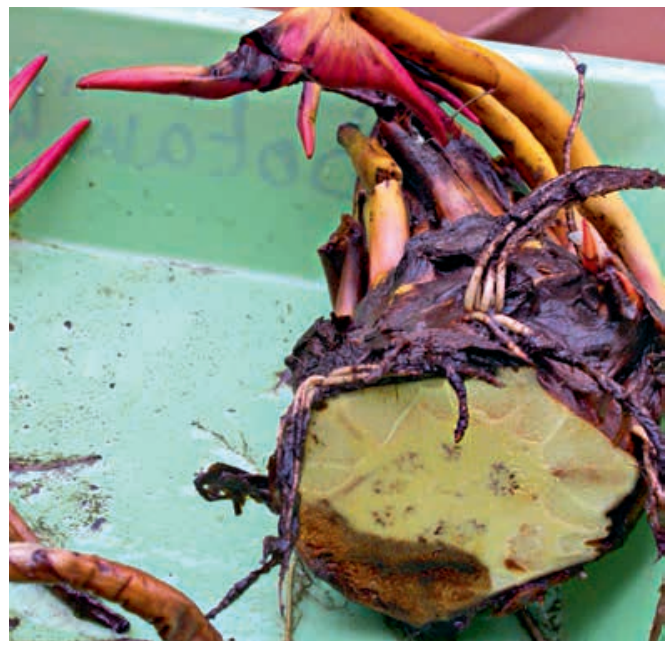

Abb. 10: Das Rhizom der Hybrid-Seerose verzweigt sich vielfach und wirkt bepelzt. (Foto: E. BRUDE)

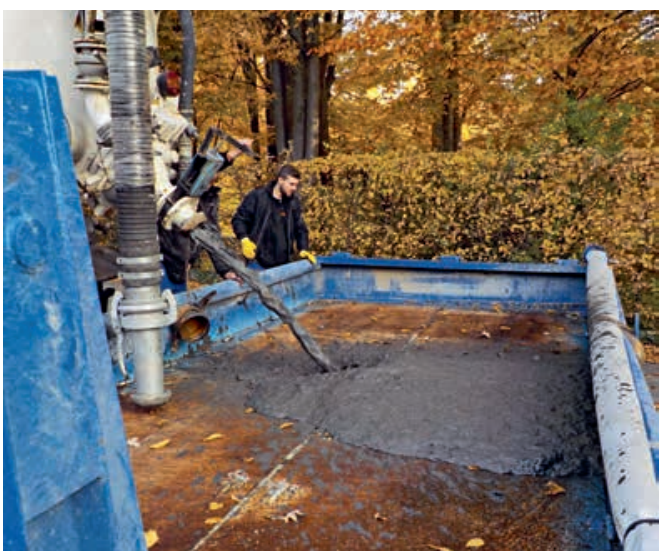

Abb. 7: Hier wird der Schlamm gelagert. Nach drei Monaten kann er fest werden. (Foto: E. BRUDE)

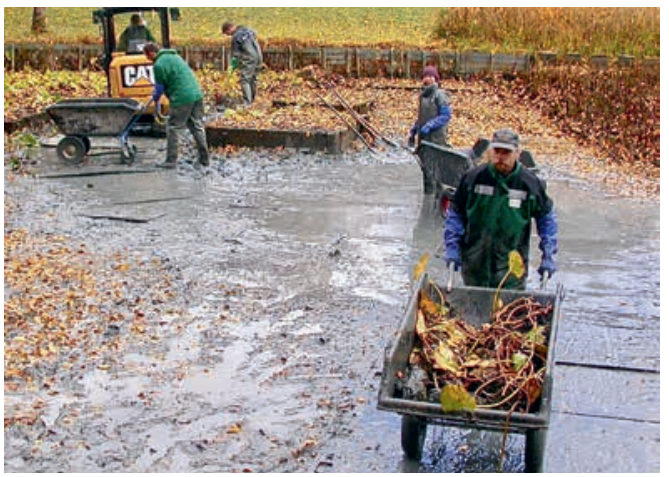

Abb. 9: Auf den Schwerlastplatten kann sich der Bagger gut bewegen. Die ausgebaggerten Seerosen werden in Schubkarren abtransportiert. (Foto: E. BRUDE)

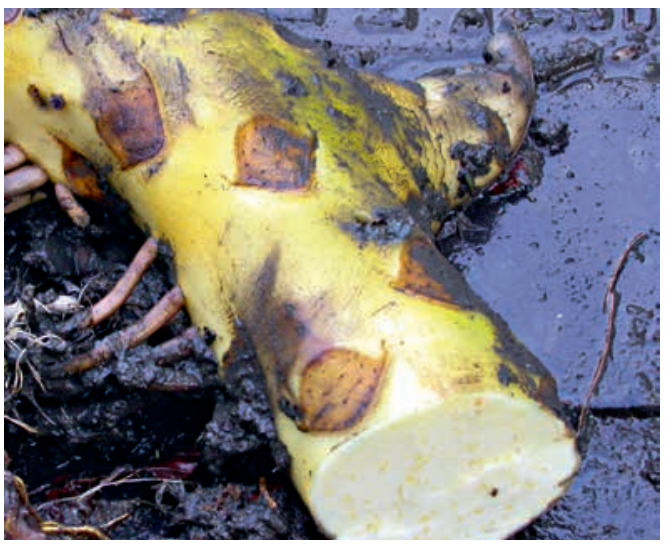

Abb. 11: Das Rhizom der Gelben Teichrose (Nuphar lutea) ist wenig verzweigt und hat eine glatte Oberfläche. Während das Rhizom der Nymphaea-Hybride im Querschnitt ein gelbes Mark mit braunen Leitbündeln zeigt, ist das NupharRhizom weiß mit gelben Leitbündeln. (Foto: E. BRUdE) 


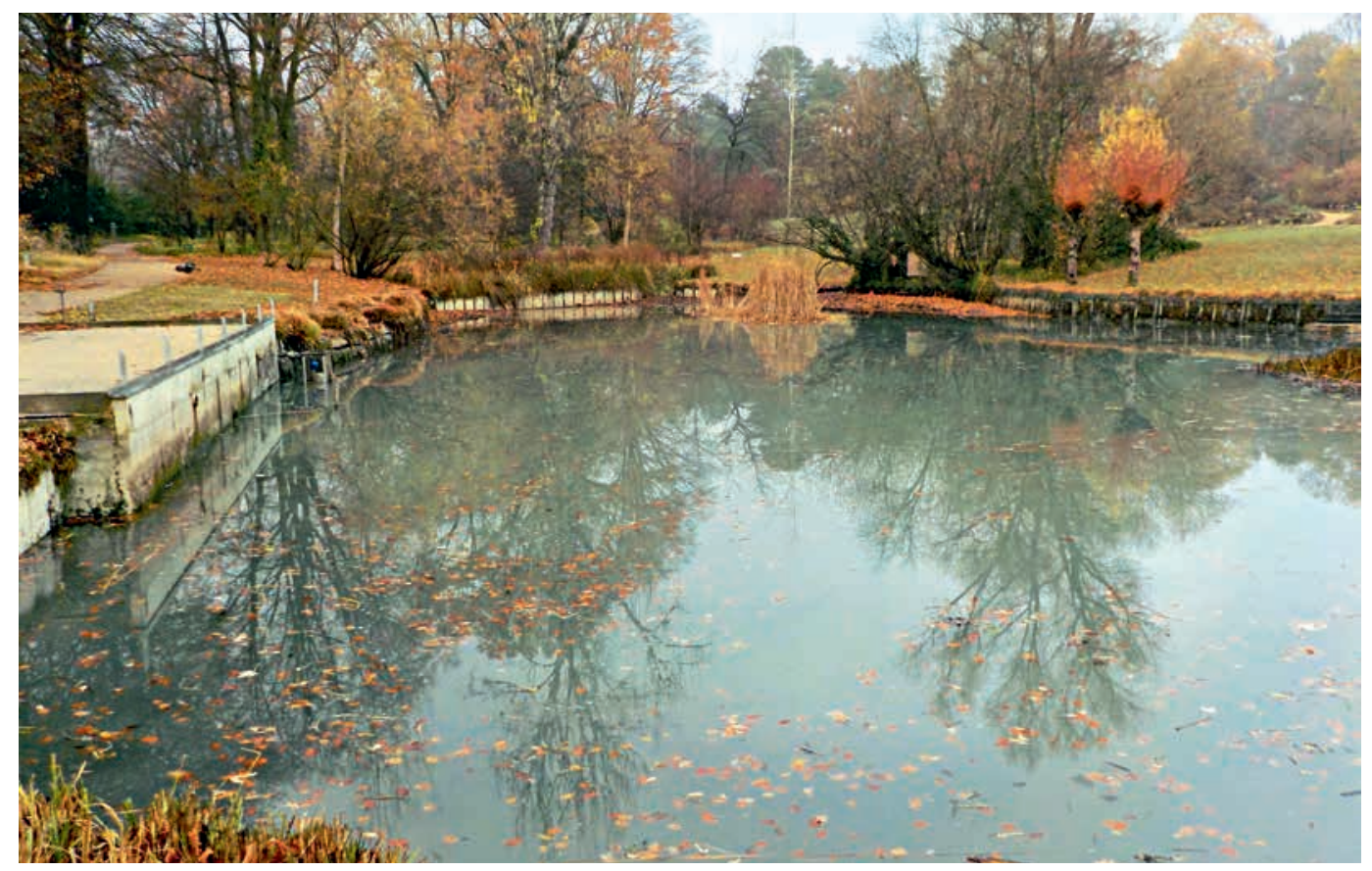

Abb. 12: Der Teich ist nun wieder mit Wasser gefüllt. (Foto: E. BRUdE)

Die flüssige Schlammfraktion war nach erfolgter Analyse zwar chemisch völlig unbedenklich, aber durch einen zu hohen Gesamtkohlenstoffgehalt (TOC-Wert: total organic carbon) für die vorgesehene Entsorgung nicht geeignet. Zunächst wurden deshalb die ca. $45 \mathrm{~m}^{3}$ Flüssigschlamm in Containern auf dem Betriebshof zwischengelagert. Später wurde in dem bestehenden großen Erdlager am Kompostplatz eine ausreichende Vertiefung geschaffen. In dieser trocknete der Schlamm bereits bis zum Frühjahr weitgehend $a b$.

Die Beseitigung der Hybrid-Seerosen mit einem Mini-Bagger (1,8 t) hat wie erhofft gut funktioniert. Damit der Bagger sich nicht in den Teichboden eingräbt und diesen beschädigt, wurden Schwerlastplatten ausgelegt. Darauf konnten auch die Schubkarren zum Abtransport der Seerosenrhizome gut fahren. Insgesamt fielen bei der Seerosenaktion etwa $30 \mathrm{~m}^{3}$ Rhizome vermischt mit festem Schlamm an, die nun ebenfalls auf dem Kompostplatz des Botanischen Gartens verrotten.

Die anschließende Wiederbefüllung des Teiches dauerte drei Tage. Die etwa 2000 Moderlieschen wurden gleich nach der Wiederbefüllung zurückgesetzt, während die etwa 1000 Jungschleien und zwei Alttiere dem Angelverein Mörfelden übergeben wurden.

\section{Neue Seerosen für den Teich}

Die wuchsstarken Seerosen-Hybriden müssen restlos entfernt werden, damit hier in Zukunft die heimische Weiße Seerose (Nymphaea alba) ohne Konkurrenzdruck als Erhaltungskultur wachsen kann. Dies ist ein längerer Prozess, denn beim Entfernen der Seerosenhybriden brechen immer wieder kleine Rhizomstücke ab. Sie verbleiben im Teichgrund und treiben später wieder aus. Im Laufe des Sommers 2019 wurden so um die hundert Stücke mit ausgetriebenen Blättern erneut entfernt. Eine Seerosen-Hybride aus einem naturnahen Gewässer zu entfernen ist kein einfaches Unterfangen.

Die für den Teich vorgesehene Weiße Seerose stammt von einem der letzten bekannten hessischen Vorkommen am Kühkopf und wurde mit Genehmigung der zuständigen Naturschutzbehörde (RP Darmstadt) seit 2012 im Botanischen Garten in einem Extrabecken kultiviert. Diese 


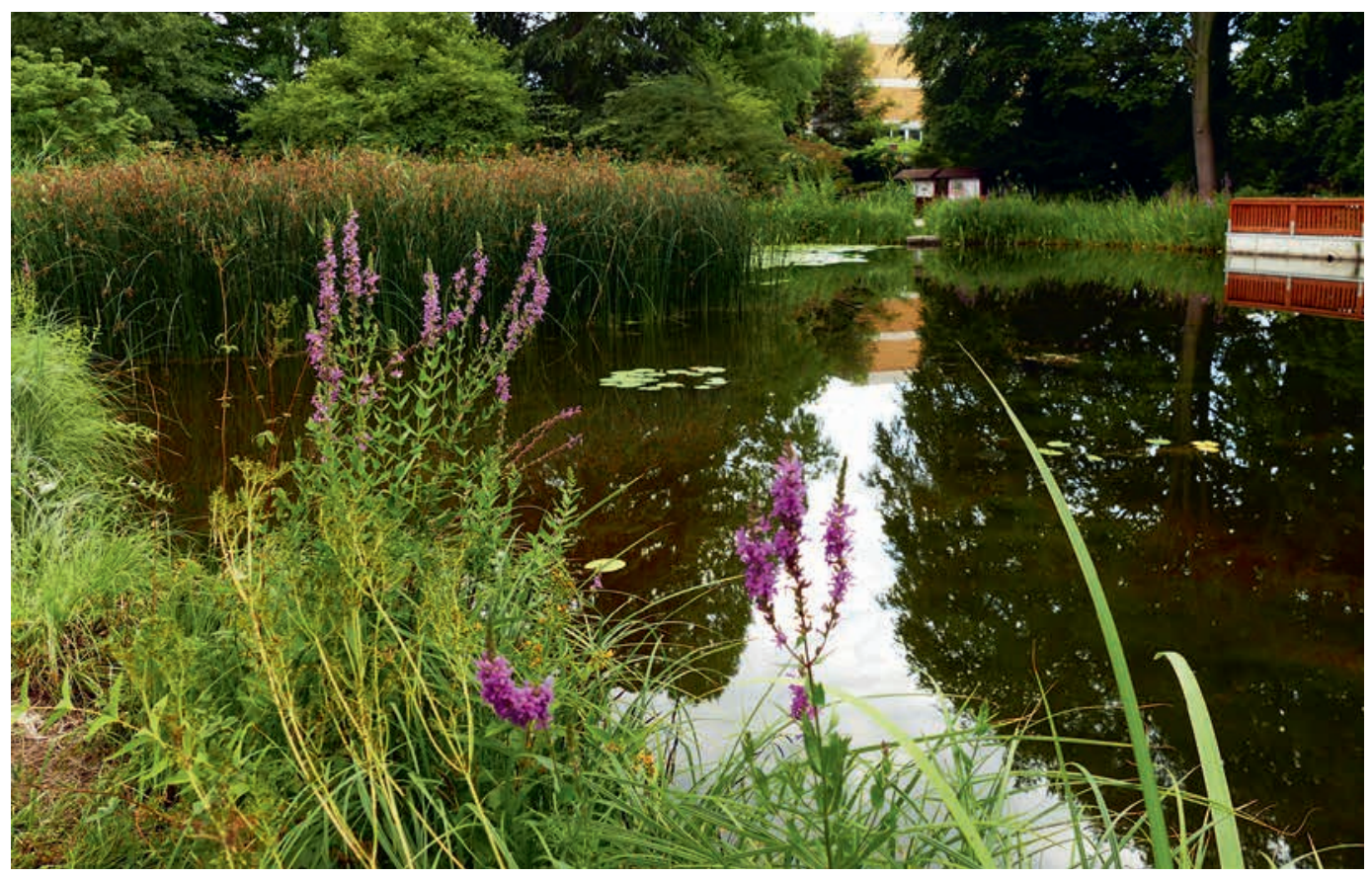

Abb. 13: Blick über den Teich im August 2020. Bei den beiden gerade nicht blühenden Seerosengruppen in der Teichmitte handelt es sich um die neuen Pflanzen von Nymphaea alba. (Foto: H. STEInecke)

Population war molekulargenetisch untersucht und als artreine einheimische Weiße Seerose erkannt worden (Nierbauer 2014). Sie wurde im Jahr 2020 erfolgreich im Teich angesiedelt.

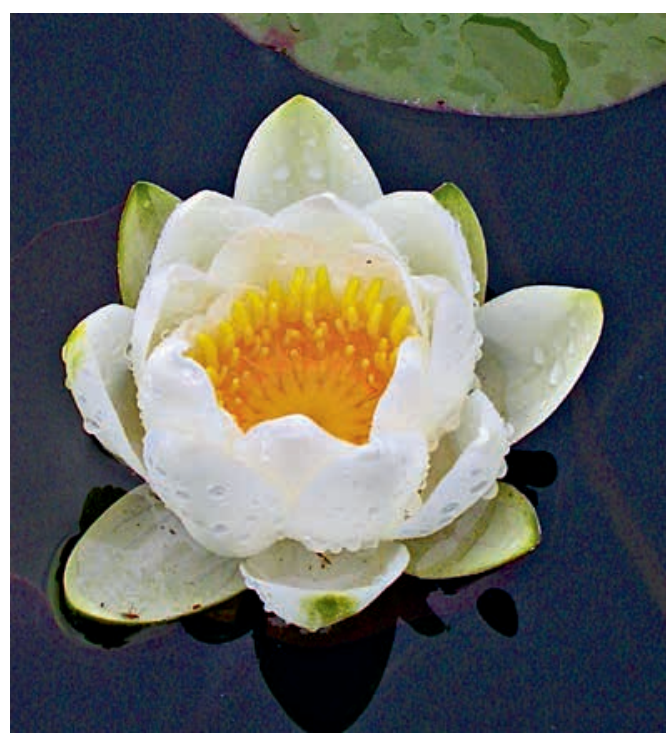

Abb. 14: Nymphaea alba in Erhaltungskultur im Botanischen Garten. (Foto: E. BRUdE)
Wir sind sehr gespannt, wie sich Nymphaea alba in ihrem neuen Gewässer entwickeln wird.

\section{Literatur}

Nierbauer, K. U., Kanz, B. \& Zizka, G. 2014: The widespread naturalisation of Nymphaea hybrids is masking the decline of Nymphaea alba in Hesse, Germany. - Flora 209: 122-130.

RP Darmstadt 2012, Az. V 53.2-2.12-R 21.6.4-6116-302

\section{Anschriften der Autorin und der Autoren}

ELKe BRUde, Freundeskreis Botanischer Garten Frankfurt am Main e. V., Siesmayerstr. 72, 60323 Frankfurt,

E-Mail: elke.brude@t-online.de

Andreas König, Botanischer Garten Frankfurt am Main, Siesmayerstr 72, 60323 Frankfurt,

E-Mail: andreas.koenig@stadt-frankfurt.de

Kai Uwe Nierbauer, Botanischer Garten Frankfurt am Main, Siesmayerstr 72, 60323 Frankfurt,

E-Mail: kai.nierbauer@stadt-frankfurt.de

Dr. Hilke Steinecke, Palmengarten Frankfurt,

Siesmayerstraße 61, 60323 Frankfurt;

E-Mail: hilke.steinecke@stadt-frankfurt.de 